\title{
Article \\ Socioeconomic Inequalities in Human Immunodeficiency Virus (HIV) Sero-Prevalence among Women in Namibia: Further Analysis of Population-Based Data
}

\author{
Michael Ekholuenetale ${ }^{1, *(\mathbb{D})}$, Herbert Onuoha ${ }^{2}$ (D), Charity Ehimwenma Ekholuenetale ${ }^{3}$, Amadou Barrow ${ }^{4}$ (D) \\ and Chimezie Igwegbe Nzoputam ${ }^{5}$ (D)
}

1 Department of Epidemiology and Medical Statistics, Faculty of Public Health, College of Medicine, University of Ibadan, Ibadan 200284, Nigeria

2 Department of Tropical Hygiene and Public Health, Medical Faculty, Heidelberg University, 69117 Heidelberg, Germany; honuoha@gmail.com

3 Department of Economics, Faculty of Social Sciences, National Open University of Nigeria, Abuja 900211, Nigeria; charityehis2016@gmail.com

4 Department of Public \& Environmental Health, School of Medicine \& Allied Health Sciences, University of The Gambia, Kanifing 3530, The Gambia; abarrow@utg.edu.gm

5 Department of Community Health, Center of Excellence in Reproductive Health Innovation (CERHI), College of Medical Sciences, University of Benin, Benin City 300001, Nigeria; chimezie.nzoputam@gmail.com

check for updates

Citation: Ekholuenetale, M.; Onuoha, H.; Ekholuenetale, C.E.; Barrow, A.; Nzoputam, C.I. Socioeconomic Inequalities in Human Immunodeficiency Virus (HIV) Sero-Prevalence among Women in Namibia: Further Analysis of Population-Based Data. Int. J. Environ. Res. Public Health 2021, 18, 9397. https://doi.org/10.3390/ ijerph18179397

Academic Editors: Tinashe Dune, Amit Arora, Zelalem Mengesha and Pranee Liamputtong

Received: 28 June 2021

Accepted: 4 September 2021

Published: 6 September 2021

Publisher's Note: MDPI stays neutral with regard to jurisdictional claims in published maps and institutional affiliations.

Copyright: (c) 2021 by the authors. Licensee MDPI, Basel, Switzerland. This article is an open access article distributed under the terms and conditions of the Creative Commons Attribution (CC BY) license (https:// creativecommons.org/licenses/by/ $4.0 /)$.
* Correspondence: mic42006@gmail.com

\begin{abstract}
Socioeconomic inequality is a major factor to consider in the prevention of human immunodeficiency virus (HIV) transmission. The aim of this study was to investigate socioeconomic inequalities in HIV prevalence among Namibian women. Data from a population-based household survey with multistage-stratified sample of 6501 women were used to examine the link between socioeconomic inequalities and HIV prevalence. The weighted HIV prevalence was $13.2 \%$ (95\% CI: 12.1-14.3\%). The HIV prevalence among the poorest, poorer, middle, richer, and richest households was $21.4 \%, 19.7 \%, 16.3 \%, 11.0 \%$, and $3.7 \%$, respectively. Similarly, $21.2 \%, 21.7 \%, 11.8 \%$, and $2.1 \%$ HIV prevalence was estimated among women with no formal education and primary, secondary, and higher education, respectively. Women from poor households (Conc. Index $=-0.258$; SE $=0.017$ ) and those with no formal education (Conc. Index $=-0.199 ; \mathrm{SE}=0.015$ ) had high concentration of HIV infection, respectively. In light of these findings, HIV prevention strategies must be tailored to the specific drivers of transmission in low socioeconomic groups, with special attention paid to the vulnerabilities faced by women and the dynamic and contextual nature of the relationship between socioeconomic status and HIV infection.
\end{abstract}

Keywords: low socioeconomic; HIV/AIDS; Namibia; concentration index; inequalities; women's health

\section{Introduction}

Globally, human immunodeficiency virus (HIV) continues to pose a serious public health challenge. Since the epidemic started, about 76 million people have been infected, and 33 million people have died from Acquired Immunodeficiency Syndrome (AIDS)related illnesses. The 2019 estimates show 38 million people living with HIV (PLHIV) worldwide, 1.7 million new HIV infections, and 690,000 AIDs-related deaths [1,2]. With a global prevalence of $0.7 \%$ among adults, the HIV burden varies significantly between regions and countries [1,2]. Despite global progress in the HIV response, Africa continues to bear the brunt of the epidemic, with a prevalence of 3.7\% [1,2]. Sub-Saharan Africa (SSA) remains disproportionately burdened with more than two-thirds (67.4\%) of the 38 million PLHIV [1,2]. In addition, SSA accounted for $57 \%$ of 1.7 million new HIV infections worldwide, and $64 \%$ of all AIDS-related deaths [1,2]. 
The 2016 Political Declaration on HIV and AIDS, adopted at the United Nations (UN) General Assembly High-Level Meeting on AIDS in June 2016, reaffirmed the 2030 Agenda for Sustainable Development. Seven of the seventeen Sustainable Development Goals (SDGs) including ending AIDS epidemic by 2030 (SDG 3.3), and those focused on poverty, hunger, education, gender equality, decent work, and reducing inequalities, are relevant to effective HIV response globally and within countries [3,4]. The concept of inequality among the populace is notable. The persistent high HIV prevalence and burden in SSA appears to be linked to income inequality [2]. Using data from 46 countries in SSA, the UN University World Institute for Development Economics Research (UNU-WIDER) demonstrated a positive relationship between HIV prevalence and income inequality after adjusting for differences across countries in levels of education, gender inequality, gross domestic production, and corruption [2,5]. This validates findings from other recent studies, reporting that social and economic disparities drive health inequalities across and within countries and that higher socioeconomic status is positively associated with better health outcomes [2,6-11].

The Southern Africa region appears to be the epicenter of the HIV epidemic, with estimated $54.5 \%$ of the 38 million PLHIV, $43 \%$ of all new HIV infections, and $43 \%$ of all AIDS-related deaths [1,2]. The leading countries with HIV prevalence include Eswatini (Swaziland), Lesotho, Botswana, South Africa, Zimbabwe, Mozambique, Namibia, Zambia, and Malawi, with prevalence ranging from $8.9 \%$ to $27 \%$ [1,2]. HIV prevalence in Namibia is among the highest in the world. Currently, Namibia has HIV prevalence of $11.5 \%$, with approximately 210,000 PLHIV and 3000 AIDS-related deaths in 2019 [2].

Women are disproportionately affected by HIV. In a recent report, women and girls accounted for 63 percent of all new HIV infections in SSA in 2020 [1]. In Namibia, HIV prevalence is higher for women $(16.9 \%)$ than men (10.9\%) [12]. Unequal gender norms limit women's and girls' voices, limit their access to education and economic resources, and inhibit civic participation, all of which contribute to women's increased HIV risk in high-HIV-prevalence environments [2]. Women are more vulnerable or most at-risk of HIV transmission and therefore become a key population of interest for HIV interventions. It is against this backdrop that this study used women as study participants.

Many HIV-related epidemiological research studies have concentrated on absolute poverty as a risk factor for HIV infection; however, studies from SSA have recently indicated that socioeconomic disparity is a bigger driver of HIV transmission than absolute poverty or wealth. Despite Namibia's high overall HIV prevalence, wide disparities in sub-national HIV prevalence, and the highly socioeconomically heterogeneous nature of its states, none of the few studies on socioeconomic inequality as a driver of HIV transmission in SSA have extensively used Namibia as a case study. Therefore, the goal of this study was to look into the differences in HIV prevalence among Namibian women based on socioeconomic status.

\section{Materials and Methods}

\subsection{Ethical Consideration}

We used population-based secondary datasets from the public domain/online that were stripped of any identifier information for this investigation. MEASURE Demographic and Health Survey (DHS)/Inner City Fund (ICF) International gave the authors permission to use the data. The DHS Program adheres to industry norms for preserving the privacy of respondents. ICF International assures that the survey complies with the Human Subjects Protection Act of the United States Department of Health and Human Services. This study did not require any additional approvals. More details about data and ethical standards are available at http:/ / goo.gl/ny8T6X (accessed on 15 June 2021).

\subsection{Data Source}

We used nationally representative cross-sectional data from the 2013 Survey of Namibia DHS. Data on 6501 women aged 15-64 years were extracted from the study individual-level 
DHS survey and analyzed. These data linked survey responses with HIV test results from biomarker data.

The Namibia DHS began in April 2012, and data gathering took place from May to September 2013. The survey's general goal was to collect demographic, socioeconomic, and health data that might be used for national and regional policymaking, planning, monitoring, and evaluation.

The specific survey's goal was to extract reliable data on HIV / AIDS, to explore the current prevalence, and to examine if progress on the issue over time could be determined. Details of the DHS sampling procedure have been previously reported [13].

\subsection{Variables Selection and Measurement}

\subsubsection{Outcome}

The dependent variable was a dichotomous indicator of HIV status positivity: a value of 1 or 0 indicated whether a respondent was seropositive (1) or negative (0), respectively.

The status of the women was determined by taking a blood sample from each of them. This study looked into the HIV status of women (positive vs. negative).

\subsubsection{Explanatory Factors}

The inclusion of these independent variables was based on the outcome of factors associated with HIV from previous studies [14-18]. The explanatory factors are presented in Table 1.

Table 1. Explanatory factors for HIV prevalence among women in Namibia.

\begin{tabular}{|c|c|}
\hline Variable & Definition \\
\hline Age & $15-19,20-24,25-29,30-34,35-39,40-44,45-49,50+$ \\
\hline Residential status & urban versus rural \\
\hline & Caprivi, Erongo, Hardap, Karas, Kavango, Khomas, Kunene, \\
\hline Region & $\begin{array}{l}\text { Ohangwena, Omaheke, Omusati, Oshana, Oshikoto, } \\
\text { Otjozondjupa }\end{array}$ \\
\hline Sex of household head & male versus female \\
\hline $\begin{array}{l}\text { Frequency of reading } \\
\text { newspaper or magazine }\end{array}$ & not at all, less than once a week, at least once a week \\
\hline Frequency of listening to radio & not at all, less than once a week, at least once a week \\
\hline Frequency of watching TV & not at all, less than once a week, at least once a week \\
\hline Smoking & no versus yes \\
\hline Health insurance coverage & not covered versus covered \\
\hline Marital status & never in union, currently in union/married, formerly in union \\
\hline Participation in labor force & not employed versus employed \\
\hline
\end{tabular}

\subsubsection{Socioeconomic Variables}

In this study, we extracted women's education and household wealth index variables as socioeconomic factors. DHS computed a household wealth index using principal component analysis (PCA). These were the variables extracted for socioeconomic measurements. Women's education and household wealth quintiles were utilized as socioeconomic status indicators in this study. Inequalities in HIV prevalence were investigated as a result of household wealth and women's educational level. Previous studies [19-23] also used women's education, household wealth, or both while investigating for socioeconomic inequalities, as these two indicators were identified as the most important measures of socioeconomic status. Women's education was categorized into groups (no formal education, primary, secondary, higher).

The household wealth index was calculated as a cumulative composite of each polled household's living standard. It was calculated using data that were simple to obtain on each of the surveyed household's specified assets. Bicycles and televisions were among the selected assets, as were the materials used to build or construct the houses, the sort of water available to the families, and their sanitary facilities. The wealth index for each 
household was calculated using principal component analysis and placed on a continuous relative wealth scale. DHS divided the questioned families into five wealth quintiles in principle, using PCA to compute the household variables. PCA has been established and validated as a tool for explaining how a population's socioeconomic status is differentiated within that population. It has also been used to cut down on the number of variables in a batch of data [24]. Each household's $z$-scores and factor loadings (factor coefficient) was determined. The loadings were multiplied by the indicator values of each household and then summed to provide the wealth index value for each household. With the standardized z-scores, the overall assigned scores of the poorest/poorer/middle/richer/richest groups were disentangled [12].

\subsection{Analytical Approach}

To account for sampling design, the Stata survey module ('svy') command was utilized. Percentage was used in the univariate test. Socio-economic inequalities were studied using the Lorenz curve and concentration index for HIV prevalence. The concentration index value is positive when HIV prevalence is higher among high socioeconomic classes. When the concentration index value is negative, it suggests that HIV prevalence is high among poor socioeconomic groups. For stratified analyses, explanatory variables were used. In HIV prevalence, the concentration index was utilized to compute the contrast $[23,25]$. Statistical significance was determined at $p<0.05$. Stata version 14 (StataCorp., College Station, TX, USA) was used for data analysis.

\section{Results}

The weighted HIV prevalence was 13.2\% (95\% CI: 12.1-14.3\%). In Table 2, the results showed increasing HIV prevalence as women advanced in age. The prevalence was higher among those in rural residence, among female headed households, among women with no media use (newspaper/magazine, radio and TV), those not covered by health insurance, and those formerly in a union and not employed, respectively. See Table 2 for the details.

Table 2. Distribution of HIV prevalence across women's characteristics in Namibia.

\begin{tabular}{|c|c|c|}
\hline Variable & $n(\%)$ & HIV Sero-Prevalence, $\%$ \\
\hline \multicolumn{3}{|l|}{ Age } \\
\hline $15-19$ & $627(9.6)$ & 2.6 \\
\hline $20-24$ & $1118(17.2)$ & 2.9 \\
\hline $25-29$ & $1072(16.5)$ & 9.0 \\
\hline $30-34$ & $924(14.2)$ & 16.9 \\
\hline $35-39$ & $866(13.3)$ & 23.0 \\
\hline $40-44$ & $758(11.7)$ & 24.0 \\
\hline $45-49$ & $592(9.1)$ & 19.8 \\
\hline $50+$ & $544(8.4)$ & 16.5 \\
\hline \multicolumn{3}{|l|}{ Residential status } \\
\hline Urban & $3487(53.6)$ & 11.2 \\
\hline Rural & $3014(46.4)$ & 16.5 \\
\hline \multicolumn{3}{|l|}{ Region } \\
\hline Caprivi & $406(6.3)$ & 24.1 \\
\hline Erongo & $630(9.7)$ & 7.9 \\
\hline Hardap & $372(5.7)$ & 9.1 \\
\hline Karas & $577(8.9)$ & 10.9 \\
\hline Kavango & $507(7.8)$ & 16.2 \\
\hline Khomas & $693(10.7)$ & 7.2 \\
\hline Kunene & $396(6.1)$ & 7.1 \\
\hline Ohangwena & $475(7.3)$ & 19.6 \\
\hline Omaheke & $392(6.0)$ & 9.4 \\
\hline Omusati & $549(8.4)$ & 23.2 \\
\hline
\end{tabular}


Table 2. Cont.

\begin{tabular}{|c|c|c|}
\hline Variable & $n(\%)$ & HIV Sero-Prevalence, $\%$ \\
\hline Oshana & $550(8.5)$ & 17.5 \\
\hline Oshikoto & $480(7.4)$ & 14.6 \\
\hline Otjozondjupa & $474(7.3)$ & 12.7 \\
\hline \multicolumn{3}{|l|}{ Sex of household head } \\
\hline Male & $2840(43.7)$ & 10.1 \\
\hline Female & $3661(56.3)$ & 16.4 \\
\hline \multicolumn{3}{|c|}{ Frequency of reading newspaper or magazine } \\
\hline Not at all & $2074(32.0)$ & 20.6 \\
\hline Less than once a week & $2105(32.4)$ & 13.7 \\
\hline At least once a week & $2309(35.6)$ & 7.4 \\
\hline \multicolumn{3}{|l|}{ Frequency of listening to radio } \\
\hline Not at all & $1072(16.5)$ & 16.0 \\
\hline Less than once a week & $1515(23.3)$ & 15.3 \\
\hline At least once a week & $3908(60.2)$ & 12.4 \\
\hline \multicolumn{3}{|l|}{ Frequency of watching TV } \\
\hline Not at all & $2950(45.8)$ & 19.4 \\
\hline Less than once a week & $869(13.4)$ & 12.0 \\
\hline At least once a week & $2650(40.8)$ & 7.8 \\
\hline \multicolumn{3}{|l|}{ Smoking } \\
\hline No & $6050(93.1)$ & 13.8 \\
\hline Yes & $451(6.9)$ & 11.5 \\
\hline \multicolumn{3}{|l|}{ Health insurance coverage } \\
\hline Not covered & $5303(81.6)$ & 15.4 \\
\hline Covered & $1197(18.4)$ & 6.1 \\
\hline \multicolumn{3}{|l|}{ Marital status } \\
\hline Never in union & $3183(49.0)$ & 10.7 \\
\hline Currently in union/married & $2645(40.7)$ & 13.1 \\
\hline Formerly in union & $673(10.4)$ & 30.0 \\
\hline \multicolumn{3}{|l|}{ Participation in labor force } \\
\hline Not employed & $3302(51.1)$ & 14.8 \\
\hline Employed & $3164(48.9)$ & 12.5 \\
\hline Total estimates & 6501 & 13.2 \\
\hline
\end{tabular}

In Table 3, the HIV prevalence among the poorest, poorer, middle, richer, and richest households was $21.4 \%, 19.7 \%, 16.3 \%, 11.0 \%$, and $3.7 \%$, respectively. The results show a decreasing pattern of HIV prevalence across women's characteristics. HIV prevalence were higher among women from low household wealth quintiles. See Table 3 for the details.

In Table 4, approximately $21.2 \%, 21.7 \%, 11.8 \%$, and $2.1 \%$ HIV prevalence was estimated among women with no formal education and primary, secondary, and higher education, respectively. The educated women had the lowest HIV prevalence, and this pattern was consistent across women's characteristics. See Table 4 for the details.

Figures 1 and 2 show household wealth and women's education inequalities for HIV prevalence in Namibia. A higher degree of inequalities is confirmed by how far the curves sag away from the line of equality (that is the red diagonal line). Figures 1 and 2 show that women from poor households and those with low formal education had a higher HIV prevalence, as the line of equality sags above the diagonal line respectively. 
Table 3. Distribution of HIV prevalence by household wealth across women's characteristics in Namibia.

\begin{tabular}{|c|c|c|c|c|c|}
\hline Variable & $\begin{array}{c}\text { Lowest } \\
\text { (Poorest) }\end{array}$ & $\begin{array}{c}\text { Second } \\
\text { Level }\end{array}$ & Middle & $\begin{array}{c}\text { Fourth } \\
\text { Level }\end{array}$ & $\begin{array}{c}\text { Highest } \\
\text { (Richest) }\end{array}$ \\
\hline \multicolumn{6}{|l|}{ Age } \\
\hline $15-19$ & 3.2 & 3.0 & 2.1 & 2.3 & 2.1 \\
\hline $20-24$ & 6.9 & 3.5 & 3.8 & 1.3 & 1.2 \\
\hline $25-29$ & 18.8 & 10.3 & 11.4 & 5.5 & 3.3 \\
\hline $30-34$ & 20.9 & 31.7 & 22.8 & 12.7 & 2.5 \\
\hline $35-39$ & 29.2 & 33.3 & 27.9 & 20.5 & 5.9 \\
\hline $40-44$ & 41.4 & 33.3 & 32.0 & 20.2 & 5.8 \\
\hline $45-49$ & 37.7 & 34.3 & 16.8 & 18.8 & 3.3 \\
\hline $50+$ & 20.6 & 21.1 & 19.4 & 16.4 & 6.3 \\
\hline \multicolumn{6}{|l|}{ Residential status } \\
\hline Urban & 29.8 & 22.3 & 17.1 & 11.6 & 3.9 \\
\hline Rural & 20.9 & 18.6 & 15.5 & 9.6 & 0.9 \\
\hline \multicolumn{6}{|l|}{ Region } \\
\hline Caprivi & 29.0 & 25.0 & 24.5 & 22.0 & 10.0 \\
\hline Erongo & 33.3 & 13.3 & 16.4 & 11.1 & 2.7 \\
\hline Hardap & 35.7 & 25.0 & 11.8 & 7.2 & 2.4 \\
\hline Karas & 11.8 & 16.7 & 19.7 & 10.5 & 3.2 \\
\hline Kavango & 15.6 & 15.7 & 16.2 & 20.3 & 13.3 \\
\hline Khomas & 20.0 & 21.9 & 15.2 & 5.8 & 1.8 \\
\hline Kunene & 4.2 & 3.3 & 13.8 & 7.3 & 5.8 \\
\hline Ohangwena & 24.3 & 21.3 & 13.6 & 9.1 & 0.0 \\
\hline Omaheke & 13.3 & 17.5 & 6.4 & 7.1 & 3.1 \\
\hline Omusati & 32.2 & 21.6 & 19.5 & 26.1 & 4.4 \\
\hline Oshana & 24.0 & 23.6 & 21.3 & 13.2 & 6.2 \\
\hline Oshikoto & 17.0 & 23.4 & 13.1 & 9.5 & 2.0 \\
\hline Otjozondjupa & 25.0 & 24.4 & 13.8 & 10.7 & 7.6 \\
\hline \multicolumn{6}{|l|}{ Sex of household head } \\
\hline Male & 14.2 & 15.7 & 14.3 & 10.4 & 1.6 \\
\hline Female & 25.6 & 22.7 & 17.7 & 11.4 & 6.4 \\
\hline \multicolumn{6}{|c|}{ Frequency of reading newspaper or magazine } \\
\hline Not at all & 23.2 & 23.1 & 19.8 & 14.1 & 14.5 \\
\hline Less than once a week & 19.7 & 16.0 & 17.1 & 12.2 & 3.8 \\
\hline At least once a week & 13.5 & 17.9 & 10.9 & 8.7 & 2.2 \\
\hline \multicolumn{6}{|c|}{ Frequency of listening to radio } \\
\hline Not at all & 21.9 & 22.1 & 14.1 & 10.0 & 3.0 \\
\hline Less than once a week & 23.4 & 19.2 & 18.6 & 11.7 & 4.0 \\
\hline At least once a week & 19.9 & 19.1 & 16.0 & 11.0 & 3.7 \\
\hline \multicolumn{6}{|c|}{ Frequency of watching TV } \\
\hline Not at all & 22.1 & 21.0 & 17.7 & 13.4 & 0.0 \\
\hline Less than once a week & 16.7 & 15.6 & 17.5 & 10.8 & 4.5 \\
\hline At least once a week & 11.8 & 12.5 & 12.4 & 10.4 & 3.6 \\
\hline \multicolumn{6}{|l|}{ Smoking } \\
\hline No & 21.7 & 19.3 & 16.5 & 11.3 & 4.0 \\
\hline Yes & 18.6 & 26.4 & 14.0 & 7.5 & 0.0 \\
\hline \multicolumn{6}{|c|}{ Health insurance coverage } \\
\hline Not covered & 21.3 & 20.0 & 16.5 & 11.5 & 5.3 \\
\hline Covered & 27.3 & 14.8 & 14.1 & 9.0 & 1.9 \\
\hline
\end{tabular}


Table 3. Cont.

\begin{tabular}{lccccc}
\hline \multicolumn{1}{c}{ Variable } & $\begin{array}{c}\text { Lowest } \\
\text { (Poorest) }\end{array}$ & $\begin{array}{c}\text { Second } \\
\text { Level }\end{array}$ & Middle & $\begin{array}{c}\text { Fourth } \\
\text { Level }\end{array}$ & $\begin{array}{c}\text { Highest } \\
\text { (Richest) }\end{array}$ \\
\hline Marital status & & & & & \\
\hline Never in union & 15.8 & 14.4 & 12.4 & 9.3 & 3.7 \\
Currently in union/married & 20.7 & 20.4 & 17.3 & 10.3 & 2.3 \\
Formerly in union & 38.9 & 42.3 & 34.1 & 23.5 & 10.6 \\
\hline Participation in labor force & & & & & \\
\hline Not employed & 22.7 & 18.1 & 15.9 & 10.0 & 3.0 \\
Employed & 18.1 & 22.8 & 16.9 & 11.6 & 3.9 \\
Total estimates & 21.4 & 19.7 & 16.3 & 11.0 & 3.7 \\
\hline
\end{tabular}

Table 4. Distribution of HIV prevalence by educational status across women's characteristics in Namibia.

\begin{tabular}{|c|c|c|c|c|}
\hline Variable & No Education & Primary & Secondary & Higher \\
\hline \multicolumn{5}{|l|}{ Age } \\
\hline 15-19 & 0.0 & 3.5 & 2.5 & 0.0 \\
\hline $20-24$ & 11.1 & 7.6 & 2.3 & 0.0 \\
\hline $25-29$ & 16.3 & 14.0 & 8.5 & 1.1 \\
\hline $30-34$ & 26.8 & 34.4 & 13.8 & 0.0 \\
\hline $35-39$ & 29.3 & 29.1 & 22.5 & 1.8 \\
\hline $40-44$ & 24.0 & 29.9 & 23.8 & 6.5 \\
\hline $45-49$ & 27.1 & 26.6 & 16.3 & 5.8 \\
\hline $50+$ & 15.3 & 18.8 & 17.4 & 4.2 \\
\hline \multicolumn{5}{|l|}{ Residential status } \\
\hline Urban & 27.5 & 19.9 & 10.1 & 1.2 \\
\hline Rural & 17.3 & 22.8 & 14.1 & 5.5 \\
\hline \multicolumn{5}{|l|}{ Region } \\
\hline Caprivi & 33.3 & 34.6 & 22.0 & 8.7 \\
\hline Erongo & 25.0 & 13.8 & 7.4 & 1.6 \\
\hline Hardap & 18.2 & 18.8 & 6.4 & 3.5 \\
\hline Karas & 10.0 & 17.1 & 10.3 & 0.0 \\
\hline Kavango & 17.1 & 16.3 & 16.2 & 12.5 \\
\hline Khomas & 11.1 & 19.1 & 8.0 & 0.6 \\
\hline Kunene & 4.9 & 9.0 & 7.9 & 0.0 \\
\hline Ohangwena & 38.2 & 32.0 & 10.8 & 10.5 \\
\hline Omaheke & 18.5 & 6.8 & 8.7 & 0.0 \\
\hline Omusati & 41.9 & 34.0 & 17.2 & 11.1 \\
\hline Oshana & 50.0 & 28.2 & 17.8 & 0.0 \\
\hline Oshikoto & 24.1 & 22.9 & 12.0 & 0.0 \\
\hline Otjozondjupa & 27.5 & 21.0 & 8.9 & 0.0 \\
\hline \multicolumn{5}{|l|}{ Sex of household head } \\
\hline Male & 15.3 & 14.3 & 9.5 & 1.1 \\
\hline Female & 27.0 & 27.4 & 13.5 & 3.2 \\
\hline \multicolumn{5}{|c|}{ Frequency of reading newspaper or magazine } \\
\hline Not at all & 20.4 & 22.2 & 19.6 & 0.0 \\
\hline Less than once a week & 26.1 & 21.0 & 12.5 & 6.7 \\
\hline At least once a week & 42.9 & 20.9 & 7.2 & 0.7 \\
\hline \multicolumn{5}{|c|}{ Frequency of listening to radio } \\
\hline Not at all & 19.8 & 22.3 & 13.8 & 0.0 \\
\hline Less than once a week & 28.2 & 21.9 & 13.4 & 3.1 \\
\hline At least once a week & 19.2 & 21.5 & 10.8 & 2.2 \\
\hline
\end{tabular}


Table 4. Cont.

\begin{tabular}{lcccc}
\hline \multicolumn{1}{c}{ Variable } & No Education & Primary & Secondary & Higher \\
\hline Frequency of watching TV & & & & \\
\hline Not at all & 22.5 & 23.2 & 17.0 & 5.3 \\
Less than once a week & 17.7 & 20.4 & 10.9 & 2.4 \\
At least once a week & 15.9 & 17.1 & 7.6 & 1.7 \\
Smoking & & & & \\
No & 22.3 & 22.3 & 12.0 & 2.2 \\
Yes & 16.4 & 15.9 & 8.4 & 0.0 \\
\hline Health insurance coverage & & & & \\
\hline Not covered & 21.4 & 22.0 & 12.9 & 2.3 \\
Covered & 14.3 & 18.4 & 6.6 & 1.5 \\
Marital status & & & 8.8 & 2.0 \\
Never in union & 26.8 & 20.3 & 12.5 & \\
Currently in union/married & 16.4 & 17.7 & 28.4 & \\
Formerly in union & 26.2 & 36.8 & & 1.8 \\
\hline Participation in labor force & & & 12.1 & 2.3 \\
\hline Not employed & 21.1 & 21.9 & 11.4 & \\
Employed & 22.0 & 21.9 & 11.8 & \\
Total estimates & 21.2 & 21.7 &
\end{tabular}

Table 5 shows the results of household wealth and women's education inequalities for HIV prevalence in Namibia. Overall, women from poor households (Conc. Index $=-0.258$; $\mathrm{SE}=0.017)$ and those with low formal education (Conc. Index $=-0.199 ; \mathrm{SE}=0.015$ ) had a higher concentration of HIV infection. Across the levels of women's characteristics, there was higher HIV prevalence among the poor. Similarly, women with low formal education status had significantly higher HIV prevalence across the levels of explanatory factors. See Table 5 below for the details.

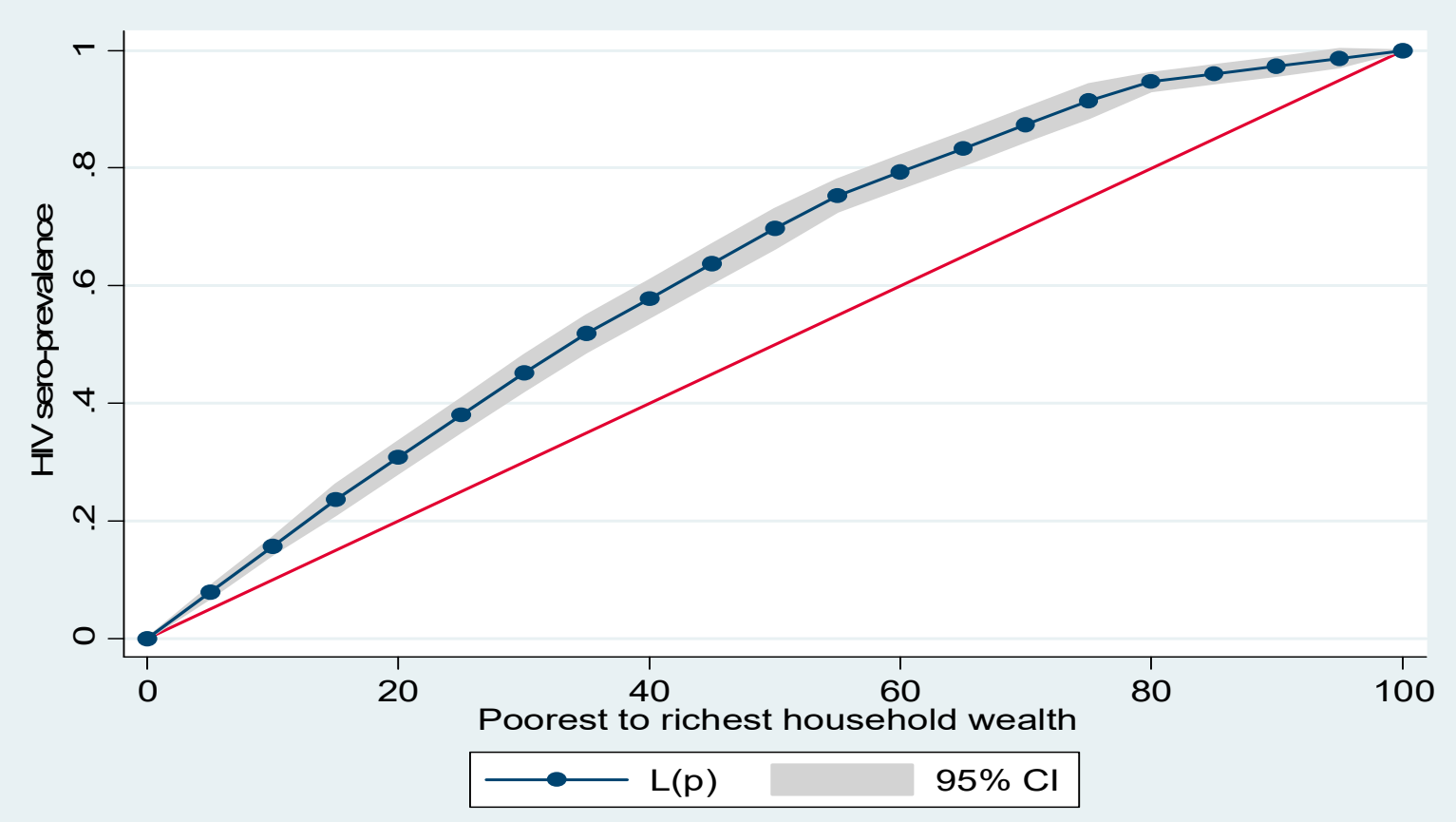

Figure 1. Lorenz curve for HIV prevalence by women's household wealth. 


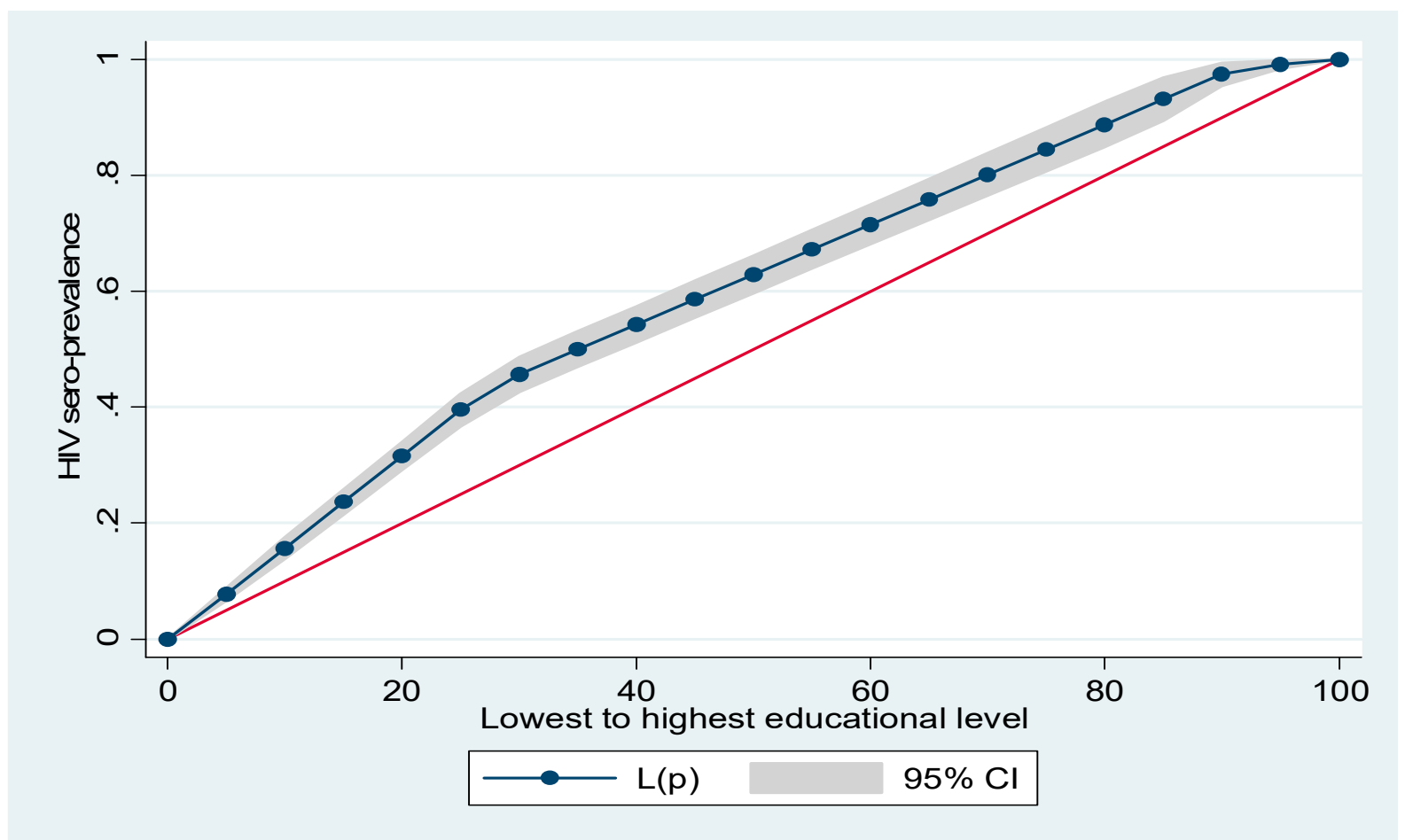

Figure 2. Lorenz curve for HIV prevalence by women's education.

Table 5. Household wealth and educational attainment inequalities in HIV prevalence among Namibian women.

\begin{tabular}{|c|c|c|c|c|}
\hline \multirow{2}{*}{ Variable } & \multicolumn{2}{|c|}{ Household Wealth Quintile } & \multicolumn{2}{|c|}{ Mother's Education } \\
\hline & Concentration Index (SE) & $p$ & Concentration Index (SE) & $p$ \\
\hline Age & & 0.279 & & $0.002 *$ \\
\hline $15-19$ & $-0.085(0.140)$ & & $-0.068(0.106)$ & \\
\hline $20-24$ & $-0.324(0.098) *$ & & $-0.342(0.077) *$ & \\
\hline $25-29$ & $-0.284(0.054) *$ & & $-0.168(0.044) *$ & \\
\hline $30-34$ & $-0.282(0.040) *$ & & $-0.249(0.033) *$ & \\
\hline $35-39$ & $-0.209(0.034) *$ & & $-0.112(0.030) *$ & \\
\hline $40-44$ & $-0.297(0.035) *$ & & $-0.095(0.033) *$ & \\
\hline $45-49$ & $-0.338(0.045) *$ & & $-0.185(0.044) *$ & \\
\hline $50+$ & $-0.165(0.054) *$ & & $-0.056(0.052)$ & \\
\hline Residential status & & $<0.001 *$ & & $<0.001 *$ \\
\hline Urban & $-0.325(0.026) *$ & & $-0.251(0.022) *$ & \\
\hline Rural & $-0.149(0.023) *$ & & $-0.120(0.021) *$ & \\
\hline Region & & $<0.001^{*}$ & & $0.001 *$ \\
\hline Caprivi & $-0.100(0.049) *$ & & $-0.122(0.040) *$ & \\
\hline Erongo & $-0.340(0.071) *$ & & $-0.188(0.059)$ * & \\
\hline Hardap & $-0.428(0.088) *$ & & $-0.272(0.077)$ * & \\
\hline Karas & $-0.288\left(0.065^{*}\right)$ & & $-0.153(0.054) *$ & \\
\hline Kavango & $0.021(0.056)$ & & $-0.013(0.053)$ & \\
\hline Khomas & $-0.488(0.071)$ * & & $-0.324(0.066)$ * & \\
\hline Kunene & $0.066(0.103)$ & & $-0.008(0.097)$ & \\
\hline Ohangwena & $-0.156(0.050) *$ & & $-0.281(0.046) *$ & \\
\hline Omaheke & $-0.272(0.087) *$ & & $-0.170(0.081) *$ & \\
\hline Omusati & $-0.099(0.043)$ * & & $-0.195(0.038) *$ & \\
\hline Oshana & $-0.194(0.051) *$ & & $-0.198(0.041) *$ & \\
\hline Oshikoto & $-0.193(0.062) *$ & & $-0.216(0.054) *$ & \\
\hline Otjozondjupa & $-0.191(0.065)$ & & $-0.278(0.058)$ * & \\
\hline Sex of household head & & 0.109 & & 0.276 \\
\hline Male & $-0.286(0.031) *$ & & $-0.176(0.028) *$ & \\
\hline Female & $-0.228(0.021)$ * & & $-0.211(0.018)^{*}$ & \\
\hline
\end{tabular}


Table 5. Cont.

\begin{tabular}{|c|c|c|c|c|}
\hline \multirow{2}{*}{ Variable } & \multicolumn{2}{|c|}{ Household Wealth Quintile } & \multicolumn{2}{|c|}{ Mother's Education } \\
\hline & Concentration Index (SE) & $p$ & Concentration Index (SE) & $p$ \\
\hline Frequency of reading newspaper or magazine & & $<0.001 *$ & & $<0.001 *$ \\
\hline Not at all & $-0.083(0.024) *$ & & $-0.028(0.023)$ & \\
\hline Less than once a week & $-0.180(0.031) *$ & & $-0.121(0.023) *$ & \\
\hline At least once a week & $-0.350(0.040) *$ & & $-0.289(0.032) *$ & \\
\hline Frequency of listening to radio & & 0.571 & & 0.517 \\
\hline Not at all & $-0.224(0.039) *$ & & $-0.162(0.036) *$ & \\
\hline Less than once a week & $-0.231(0.034) *$ & & $-0.185(0.030) *$ & \\
\hline At least once a week & $-0.266(0.023) *$ & & $-0.207(0.020) *$ & \\
\hline Frequency of watching TV & & $<0.001 *$ & & $<0.001 *$ \\
\hline Not at all & $-0.084(0.021) *$ & & $-0.086(0.019) *$ & \\
\hline Less than once a week & $-0.211(0.051) *$ & & $-0.187(0.042) *$ & \\
\hline At least once a week & $-0.250(0.035) *$ & & $-0.233(0.030) *$ & \\
\hline Smoking & & $0.019 *$ & & 0.958 \\
\hline No & $-0.248(0.018) *$ & & $-0.202(0.015) *$ & \\
\hline Yes & $-0.408(0.071) *$ & & $-0.199(0.069) *$ & \\
\hline Health insurance coverage & & $<0.001 *$ & & $<0.001 *$ \\
\hline Not covered & $-0.190(0.018) *$ & & $-0.148(0.016) *$ & \\
\hline Covered & $-0.446(0.057) *$ & & $-0.304(0.056) *$ & \\
\hline Marital status & & $0.031 *$ & & $<0.001 *$ \\
\hline Never in union & $-0.207(0.029) *$ & & $-0.245(0.023) *$ & \\
\hline Currently in union/married & $-0.303(0.028) *$ & & $-0.151(0.026) *$ & \\
\hline Formerly in union & $-0.196(0.032) *$ & & $-0.073(0.031) *$ & \\
\hline Participation in labor force & & 0.187 & & 0.137 \\
\hline Not employed & $-0.235(0.023) *$ & & $-0.175(0.021) *$ & \\
\hline Employed & $-0.281(0.026) *$ & & $-0.220(0.022) *$ & \\
\hline Total estimates & $-0.258(0.017) *$ & & $-0.199(0.015) *$ & \\
\hline
\end{tabular}

* Significant at $p<0.05 ; S E$, standard error; $p=$ comparing concentration indices across the levels of a variable.

\section{Discussion}

We found a high HIV prevalence among Namibian women (13.2 percent). This is in line with reports of HIV prevalence in the Southern Africa region, where HIV prevalence has remained the highest worldwide [26]. In a previous study, HIV prevalence in Namibians over the age of 12 years was 11.8 percent in 2006/7 and 14.6 percent in 2009 [27]. Similarly, there are staggering reports of HIV prevalence among the adult population in Eswatini (27.3\%), Lesotho (23.6\%), South Africa (20.4\%), Botswana (20.3\%), Zimbabwe (12.7\%), Mozambique (10.3\% and 12.6\%), and Zambia (11.3\%) [18,28]. Despite efforts to reduce HIV transmission over time, the rate of reduction in incidence, combined with existing contextual interventions, indicates that Namibia is still a long way from meeting the 2030 global targets for reducing HIV incidence and mortality.

HIV increases when girls are denied the right to make decisions about their sexual and reproductive health and well-being. Specific intervention must target gender inequality. That is why we must strengthen our advocacy, sensitization, and behavior change communication to really discuss issues of sexual and reproductive health and how communities can best be engaged in promoting the rights of women and girls to make the right decisions regarding their sexual and reproductive health. It is possible that previous interventions have not looked at the vulnerability of women to HIV infection with a view to designing female-focused interventions.

In addition, the results show that HIV infection is more prevalent among poor and uneducated women. These findings support the current global views on the relationship between low socioeconomic status and poor health outcomes, such as HIV infection [29-31]. The implications of the results could be that the poor and those with low educational status may be facing the dual problems of high vulnerability and a lack of possibilities to make better sexual health choices (such as access to information on prevention, testing, and 
counseling for HIV infection) as a result of low socioeconomic status. High HIV prevalence has been significantly associated with relative economic prospects, referred to as "relative wealth" by Fox [31]. In a previous study using DHS data from 20 countries in SSA, Magadi [29] found similar results, where the urban poor had greater risks of HIV infection than their non-poor counterparts.

Several measures to decrease HIV infection have been prompted by the growing HIV prevalence. For example, the number of HIV-positive patients receiving antiretroviral therapy (ART) in Namibia increased from 10,200 in 2004 [32] to 166,000 in 2016 [33]. On the other hand, the country has set a target to reduce new HIV infections by $75 \%$ by scaling up evidence-based interventions such as viral suppression through ART for all PLHIV and pre-exposure prophylaxis (PrEP) in high-burden areas [34]. However, no intervention has included specific designs targeted at women with low socioeconomic status, who were identified in this study as a key population. This evidence of HIV disproportionately infecting poor and uneducated women is timely and paramount for future HIV intervention designs. For example, interventions that include women's empowerment, decision making power, girl-child education, and women's autonomy could positively influence health care service uptake, including HIV prevention in Namibia.

Key interventions such as free HIV screening or testing and counseling and treatment for HIV positive women should be designed and supported by the government and other stakeholders in the healthcare system. This will encourage more women, especially the disadvantaged, to participate in HIV prevention, control, and treatment practices. In addition, it will be necessary for the government to enroll low income women who are HIV positive in a special financial support scheme. Such scheme could be targeted at providing economic aid to the disadvantaged women and at reducing their HIV burden. Importantly, such strategy would be useful in creating a registry for HIV positive women, which would serve as an avenue for enrolling them for ART, following-up their progress, and reducing the HIV transmission rate. Furthermore, specific messages targeted to improve awareness and knowledge of HIV among low income women, the uneducated, or those in hard-toreach communities would be helpful in the fight against HIV.

\section{Strength and Limitations}

The findings of this study can be applied to all women in Namibia because they are based on nationally representative data. However, due to the use of secondary data, it was impossible to quantify potential covariates such as the use of alcohol, hard drugs, and engagement in extramarital relationships, as well as other endogenous factors. Underrepresentation of low income women is probable in the DHS dataset. It is possible that low income women were under-represented in the survey, as some face illiteracy and perhaps could not fill out the survey or may have limited ability to accurately report their circumstances. Moreover, recruitment of the homeless or other vulnerable groups could be a major factor responsible for the under-representation of low income women. Additionally, DHS did not collect data on household income or expenditure, which are the traditional indicators used to measure wealth. The assets-based wealth index used here is only a proxy indicator for household economic status, and it does not always produce results similar to those obtained from direct measurements of income and expenditure when such data are available or can be collected reliably.

\section{Conclusions}

This study revealed high HIV prevalence among Namibian women. Furthermore, poor and uneducated Namibian women demonstrated the highest prevalence of HIV infection. These findings add to the body of knowledge about the link between HIV and socioeconomic status. HIV prevention strategies must be targeted at the vulnerabilities faced by low socioeconomic status women and the dynamic and contextual nature of the relationship between low socioeconomic status and HIV infection. 
Author Contributions: M.E. conceived and designed the study, performed data analysis, wrote the results, and discussed the findings. All authors reviewed the literature, discussed the findings, and provided substantial input and ideas into all drafts of the paper. All authors have read and agreed to the published version of the manuscript.

Funding: The technical support throughout the survey program was made possible by the Inner City Fund (ICF) with funds from the United States Agency for International Development (USAID). However, no funding or sponsorship was received for this study or for publication of this article.

Institutional Review Board Statement: Ethics approval for this study was not required since the authors used secondary data available in the public domain. Thus, IRB review is not applicable in this study. More details regarding DHS data and ethical standards are available at: http:/ / dhsprogram. com/data/available-datasets.cfm (accessed on 15 June 2021).

Informed Consent Statement: The Demographic and Health Survey is a de-identified open-source dataset. Therefore, the requirement of consent for publication is not applicable.

Data Availability Statement: Data for this study were sourced from Namibia Demographic and Health Surveys (DHS), which are available here: http:/ / dhsprogram.com/data/available-datasets. cfm (accessed on 15 June 2021).

Acknowledgments: The authors appreciate the MEASURE DHS project for the approval to use and access to the original data.

Conflicts of Interest: The authors declare that the research was conducted in the absence of any commercial or financial relationships that could be construed as a potential conflicts of interest.

\section{References}

1. Global HIV \& AIDS Statistics-2020 Fact Sheet. Available online: https://www.unaids.org/en/resources/fact-sheet (accessed on 6 August 2020).

2. Global AIDS Update. 2020-Seizing the Moment-Tackling Entrenched Inequalities to End Epidemics. 2020. Available online: https:/ / www.unaids.org/sites/default/files/media_asset/2020_global-aids-report_en.pdf (accessed on 15 June 2021).

3. United Nations. Political Declaration on HIV and AIDS: On the Fast Track to Accelerating the Fight against HIV and to Ending the AIDS Epidemic by 2030. 22 June 2016. Available online: https:/ / www.unaids.org/en/resources/documents/2016/2016political-declaration-HIV-AIDS (accessed on 15 August 2020).

4. United Nations. Transforming Our World: The 2030 Agenda for Sustainable Development. 21 October 2015. Available online: / resources/transforming-our-world-2030-agenda-sustainable-development (accessed on 15 August 2020).

5. United Nations University World Institute for Development Economics Research. UNU-WIDER: World Income Inequality Database-WIID. In: UNU-WIDER [Internet]. 17 December 2019. Available online: https:/ / www.wider.unu.edu/database/wiid (accessed on 16 August 2020).

6. Hajizadeh, M.; Sia, D.; Heymann, S.J.; Nandi, A. Socioeconomic inequalities in HIV/AIDS prevalence in sub-Saharan African countries: Evidence from the Demographic Health Surveys. Int. J. Equity Health 2014, 13, 18. [CrossRef] [PubMed]

7. Niessen, L.W.; Mohan, D.; Akuoku, J.K.; Mirelman, A.J.; Ahmed, S.; Koehlmoos, T.P. Tackling socioeconomic inequalities and non-communicable diseases in low-income and middle-income countries under the Sustainable Development agenda. Lancet 2018, 391, 2036-2046. [CrossRef]

8. World Health Organization. World Health Statistics 2020: Monitoring Health for the SDGs, Sustainable Development Goals; World Health Organization: Geneva, Switzerland, 2020. Available online: http://www.who.int/gho/publications/world_health_ statistics/2020/en/ (accessed on 2 August 2020).

9. Pons-Duran, C.; González, R.; Quintó, L.; Munguambe, K.; Tallada, J.; Naniche, D.; Sacoor, C.; Sicuri, E. Association between HIV infection and socio-economic status: Evidence from a semirural area of southern Mozambique. Trop. Med. Int. Health 2016, 21, 1513-1521. [CrossRef] [PubMed]

10. Bunyasi, E.W.; Coetzee, D.J. Relationship between socioeconomic status and HIV infection: Findings from a survey in the Free State and Western Cape Provinces of South Africa. BMJ Open 2017, 7, e016232. [CrossRef] [PubMed]

11. WHO. State of Inequality —Reproductive, Maternal, Newborn and Child Health; World Health Organization: Geneva, Switzerland, 2015. Available online: https:/ / www.who.int/docs/default-source/gho-documents/health-equity/state-of-inequality/stateof-inequality-reproductive-maternal-new-born-and-child-health.pdf?sfvrsn=f4034289_2 (accessed on 15 June 2021).

12. The Nambia Ministry of Health and Social Services (MoHSS) and ICF International. 2014. The Namibia Demographic and Health Survey 2013. Windhoek, Namibia, and Rockville, Maryland, USA: MoHSS and ICF International. Available online: https:/ / dhsprogram.com/pubs/pdf/FR298/FR298.pdf (accessed on 15 June 2021).

13. Corsi, D.J.; Neuman, M.; Finlay, J.E.; Subramanian, S. Demographic and health surveys: A profile. Int. J. Epidemiol. 2012, 41, 1602-1613. [CrossRef] [PubMed] 
14. Jewkes, R.; Dunkle, K.; Nduna, M.; Levin, J.; Jama, N.; Khuzwayo, N.; Koss, M.; Puren, A.; Duvvury, N. Factors associated with HIV sero-status in young rural South African women: Connections between intimate partner violence and HIV. Int. J. Epidemiol. 2006, 35, 1461-1468. [CrossRef]

15. Mabaso, M.; Sokhela, Z.; Mohlabane, N.; Chibi, B.; Zuma, K.; Simbayi, L. Determinants of HIV infection among adolescent girls and young women aged 15-24 years in South Africa: A 2012 population-based national household survey. BMC Public Health 2018, 18, 183. [CrossRef] [PubMed]

16. Singh, R.K.; Patra, S. What Factors Are Responsible for Higher Prevalence of HIV Infection among Urban Women than Rural Women in Tanzania? Ethiop. J. Health Sci. 2015, 25, 321-328. Available online: https://www.ncbi.nlm.nih.gov/pmc/articles/ PMC4762970/ (accessed on 15 June 2021). [CrossRef] [PubMed]

17. Bärnighausen, T.; Hosegood, V.; Timaeus, I.M.; Newell, M.-L. The socioeconomic determinants of HIV incidence: Evidence from a longitudinal, population-based study in rural South Africa. AIDS Lond. Engl. 2007, 21, S29-S38. [CrossRef]

18. Ekholuenetale, M. Seroprevalence and Determinants of Human Immunodeficiency Virus Infection among Women of Reproductive Age in Mozambique: A Multilevel Analysis. Infect. Dis. Ther. 2020, 9, 881-900. [CrossRef] [PubMed]

19. Szklarska, A.; Jankowska, E.A. Independent effects of social position and parity on body mass index among Polish adult women J. Biosoc. Sci. 2003, 35, 575-583. [CrossRef] [PubMed]

20. Walters, S.; Suhrcke, M. Socioeconomic inequalities in health and health care access in central and eastern Europe and the CIS. WHO Eur. Off. Invest. Health Dev. 2005, 50, 8-11.

21. Bado, A.R.; Sathiya Susuman, A. Women's Education and Health Inequalities in Under-Five Mortality in Selected Sub-Saharan African Countries, 1990-2015. Carpenter DO, editor. PLoS ONE 2016, 11, e0159186. [CrossRef] [PubMed]

22. Ekholuenetale, M.; Nzoputam, C.I.; Barrow, A. Prevalence and Socioeconomic Inequalities in Eight or More Antenatal Care Contacts in Ghana: Findings from 2019 Population-Based Data. Int. J. Womens Health 2021, 13, 349-360. [CrossRef] [PubMed]

23. Ekholuenetale, M.; Tudeme, G.; Onikan, A.; Ekholuenetale, C.E. Socioeconomic inequalities in hidden hunger, undernutrition, and overweight among under-five children in 35 sub-Saharan Africa countries. J. Egypt Public Health Assoc. 2020, 95, 9. [CrossRef] [PubMed]

24. Vyas, S.; Kumaranayake, L. Constructing socio-economic status indices: How to use principal components analysis. Health Policy Plan 2006, 21, 459-468. [CrossRef]

25. Uthman, O.A. Using extended concentration and achievement indices to study socioeconomic inequality in chronic childhood malnutrition: The case of Nigeria. Int. J. Equity Health 2009, 8, 22. [CrossRef] [PubMed]

26. Frank, T.D.; Carter, A.; Jahagirdar, D.; Biehl, M.H.; Douwes-Schultz, D.; Larson, S.L.; Arora, M.; Dwyer-Lindgren, L.; Steuben, K.M.; Abbastabar, H.; et al. Global, regional, and national incidence, prevalence, and mortality of HIV, 1980-2017, and forecasts to 2030, for 195 countries and territories: A systematic analysis for the Global Burden of Diseases, Injuries, and Risk Factors Study 2017. Lancet HIV. 2019, 6, e831-e859. [CrossRef]

27. Aulagnier, M.; Janssens, W.; Beer, I.D.; van Rooy, G.; Gaeb, E.; Hesp, C. Incidence of HIV in Windhoek, Namibia: Demographic and Socio-Economic Associations. PLoS ONE. 2011, 6, 9. [CrossRef] [PubMed]

28. World Health Organization. Prevalence of HIV among Adults Aged 15 to 49 Estimates by Country; WHO: Geneva, Switzerland, 2018. Available online: https:/ / apps.who.int/gho/data/node.main.622?lang=en (accessed on 25 May 2020).

29. Magadi, M.A. The disproportionate high risk of HIV infection among the urban poor in sub-Saharan Africa. AIDS Behav. 2013, 17, 1645-1654. [CrossRef]

30. Piot, P.; Greener, R.; Russell, S. Squaring the Circle: AIDS, Poverty, and Human Development. PLoS Med. 2007, 4, 5. [CrossRef] [PubMed]

31. Fox, A.M. The HIV-poverty thesis re-examined: Poverty, wealth or inequality as a social determinant of HIV infection in sub-Saharan Africa? J. Biosoc. Sci. 2012, 44, 459-480. [CrossRef] [PubMed]

32. AIDSinfo: UNAIDS. 2008. Estimates and Projections of the Impact of HIV/AIDS in Namibia. Available online: https://data. unaids.org/pub/report/2008/20080915_namibia_estimates_2008_en.pdf (accessed on 15 June 2021).

33. Maher, A.D.; Nakanyala, T.; Mutenda, N.; Banda, K.M.; Prybylski, D.; Wolkon, A.; Jonas, A.; Sawadogo, S.; Ntema, C.; Chipadze, M.R.; et al. Rates and Correlates of HIV Incidence in Namibia's Zambezi Region from 2014 to 2016: Sentinel, Community-Based Cohort Study. JMIR Public Health Surveill. 2020, 6, e17107. [CrossRef] [PubMed]

34. The United States President's Emergency Plan for AIDS Relief US Embassy in Namibia. 2017. Namibia Country Operational Plan Strategic Direction Summary, 2017. Available online: https://na.usembassy.gov/wp-content/uploads/sites/132/PEPFARStrategic-Direction-Summary-2017.pdf (accessed on 15 June 2021). 